\title{
In vitro adherence of Staphylococcus pseudintermedius to canine corneocytes is influenced by colonization status of corneocyte donors
}

\author{
Narayan Chandra Paul ${ }^{1 *}$, Francesca Latronico ${ }^{1}$, Arshnee Moodley ${ }^{1}$, Søren Saxmose Nielsen², Peter Damborg \\ and Luca Guardabassi ${ }^{1}$
}

\begin{abstract}
The current knowledge of in vitro adherence of Staphylococcus pseudintermedius to canine corneocytes is limited to comparative analyses between strains, staphylococcal species or corneocytes collected from different breeds, body sites and hosts. However, the role played by colonization status of corneocyte donors remains unknown. The aim of this study was to evaluate the adherence properties of commensal S. pseudintermedius strains to corneocytes collected from dogs with different colonization status. For this purpose, corneocytes were collected from five dogs that were classified as persistently colonized (D1 and D2), intermittently colonized (D3 and D4) or non-colonized (D5) on the basis of the results of a previous longitudinal study. Adherence to corneocytes originating from each of the five dogs was assessed by an in vitro adhesion assay using four genetically unrelated strains isolated from the colonized dogs (S1 to S4). Irrespective of their host of origin, all strains adhered significantly better to corneocytes from D1 and D2 than to corneocytes from D3, D4 and D5 $(P<0.0001)$. The mean count of cells adhering to corneocytes from persistently colonized dogs was on average three times higher than the mean count using corneocytes from the other dogs. A significant difference between strains was only observed for one straincorneocyte combination (S2-D4), indicating that S. pseudintermedius adherence to corneocytes is driven by host factors and only marginally influenced by strain factors. This finding has important implications for understanding and preventing S. pseudintermedius skin colonization and infection.
\end{abstract}

\section{Introduction}

Staphylococcus pseudintermedius is an opportunistic pathogen that resides on the skin and mucosae of dogs and other members of the Canidae. The percentage of $S$. pseudintermedius carriage reported in the scientific literature ranges between 46 and $92 \%$ among healthy dogs [1]. We have previously shown that most dogs are either persistent or intermittent carriers, and very few individuals are non-carriers over a period of one year [2].

Staphylococcal adherence to corneocytes is a crucial step for skin colonization and subsequent infection. Previous

\footnotetext{
* Correspondence: napa@sund.ku.dk

'Department of Veterinary Disease Biology, Faculty of Health and Medical Sciences, University of Copenhagen, Stigbøjlen 4, 1870 Frederiksberg C, Denmark

Full list of author information is available at the end of the article
}

studies of S. pseudintermedius adhesion to canine corneocytes mainly focused on differences between strains, staphylococcal species, dog breeds, body sites and disease status [3-5]. S. pseudintermedius adheres better to corneocytes from dogs suffering atopic dermatitis or belonging to specific breeds, and adherence to canine corneocytes by this species is greater than for other staphylococcal species typically associated with humans such as S. aureus and S. hominis [3,5]. Measurement of adherence is influenced by incubation conditions and bacterial concentrations used in the laboratory assay but no significant difference has been found between $S$. pseudintermedius isolates from pyoderma lesions and isolates from healthy dogs, suggesting a lack of correlation between virulence and in vitro adherence to canine corneocytes [4]. However, none of these studies

\section{Ciomed Central}


considered the possible role played by the colonization status of the dogs from which corneocytes were collected. In order to fill this knowledge gap, the aim of this study was to evaluate the adherence properties of commensal S. pseudintermedius strains to corneocytes collected from dogs with different colonization status. In vitro adherence was assessed using strains and corneocytes collected from persistently colonized, intermittently colonized and non-colonized dogs from a previous longitudinal study.

\section{Materials and methods}

\section{Selection of dogs}

Five dogs from a previous longitudinal study [2] were included in this study: two persistent carriers (D1, D2), two intermittent carriers (D3 and D4) and one noncarrier (D5). The dogs were classified into different carriage groups on the basis of the analysis of nine samples collected over a period of six months. Dogs were defined as persistent carriers (positive for S. pseudintermedius at all sampling times), intermittent carriers (positive in at least one sample) and non-carriers (negative at all sampling times). The colonization status of the dogs was confirmed when corneocytes were collected, approximately nine months after the end of the longitudinal study; S. pseudintermedius was isolated from both the mouth and the perineum of D1, D2 and D3, only from the perineum of $\mathrm{D} 4$, and from none of the two body sites of D5. Dogs were defined as healthy by physical examination and had no history of antimicrobial therapy and skin infection in the last six months prior to collection of corneocytes. Data on breed, sex, age and colonization status of the five dogs are summarized in Table 1.

\section{Collection of corneocytes}

Corneocytes were collected by veterinary staff during August 2012, after obtaining informed consent from the owners. Corneocytes were collected from the inner pinna of the ear by applying adhesive discs. Before collection, surface debris was removed by applying five successive adhesive tape strips (Sellotape ${ }^{\oplus}$ Original, Winsford, Cheshire, UK). Corneocytes were collected by pressing $25-\mathrm{mm}$ diameter adhesive discs (Dsquame ${ }^{\curvearrowleft}, \mathrm{CuDerm}$ Corporation, Dallas, TX, USA) to the cleaned area on both ears. The discs were then carefully removed to obtain confluent corneocyte layers and placed in sterile petri dishes. From each dog, 12-16 corneocyte samples were collected at the same time. Immediately after collection, all the corneocyte samples were transferred to the laboratory and stored at $4{ }^{\circ} \mathrm{C}$. All slides were examined by light microscopy, and only slides with confluent corneocytes were included in the experiment.

\section{Bacterial strains}

The four $S$. pseudintermedius strains used in this study (S1, S2, S3 and S4) were previously isolated from dogs D1, D2, D3 and D4 [2]. All strains were genetically distinct by pulsed-field gel electrophoresis [6] and multilocus sequence typing [7]. The genetic characteristics of the strains are described in Table 1.

\section{Corneocytes adherence assay}

The four bacterial strains were tested with all five dogs' corneocytes. The adhesion assay was done according to Moodley et al. [8] with minor modifications in the centrifugation condition (3000 rpm for $3 \mathrm{~min}$ ) and the adjusted $\mathrm{OD}_{600}$ bacterial suspension $\left((\mathrm{OD})_{600}=0.15(\sim 7 \times\right.$ $\left.10^{7} \mathrm{CFU} / \mathrm{mL}\right)$ ). Discs were examined manually using light microscopy to count adhered bacteria at $\times 1000$ magnification on a Axioplan II epifluorescence microscope (Zeiss, Oberkochen, Germany) and a Zeiss AxioCam digital camera. Ten microscopic field images with good confluent corneocytes were selected and used to count the adhered bacteria. All experiments were done in duplicate except for dog D1 and strain S3 because there were not sufficient slides with confluent corneocytes. One corneocytes disc per individual was incubated with PBS only and included as a negative control. The adhesion assay for each strain was done at the same time and performed by the same person to avoid variations in counting adherent bacteria.

\section{Statistical analysis}

Descriptive statistics were computed as the lower quartile, the median and the upper quartile for each bacterial strain adhered with each dog's corneocytes. Then, the mean adhered bacterial count of the persistent carrier dog corneocytes was compared to the mean count from non-persistent dogs with a negative binomial regression

Table 1 Data of the five dogs from which strains and corneocytes were collected

\begin{tabular}{|c|c|c|c|c|c|c|c|}
\hline Dog ID & Breed & Age (months) & Sex & S. pseudintermedius carriage status & Strain & MLST type & PFGE type \\
\hline D1 & Labrador retriever & 25 & Male & Persistent carrier & S1 & ST165 & Distinct \\
\hline D2 & German shepherd & 69 & Neutered female & Persistent carrier & S2 & ST138 & Distinct \\
\hline D3 & Labrador retriever & 39 & Female & Intermittent carrier & S3 & New ST & Distinct \\
\hline D4 & Border collie & 28 & Male & Intermittent carrier & S4 & ST20 & Distinct \\
\hline D5 & Saluki & 64 & Female & Non-carrier & - & NA & NA \\
\hline
\end{tabular}

MLST multi-locus sequence typing, ST sequence type, PFGE pulsed-field gel electrophoresis, NA not applicable. 
model using the Genmod procedure in SAS version 9.3 (SAS Institute, Cary, NC, USA). The model was also used to determine if there was any preferential binding of a particular strain to corneocytes from individual dogs, and if a particular strain adhered better to corneocytes in general.

\section{Results}

Boxplots for adherence values of the four S. pseudintermedius strains to the corneocytes from the five dogs are illustrated in Figure 1. All S. pseudintermedius strains adhered significantly better to the corneocytes from both persistent carriers (D1 and D2) in comparison to those from the two intermittent carriers (D3 and D4) and the non-carrier (D5) in the negative binomial model $(P<0.0001)$. The mean count of bacteria adhering to corneocytes from D1 and D2 was 224 cells per microscope field (95\% confidence interval: 193-258), whereas the mean count using corneocytes from non-persistent carriers was only 74 cells per field (95\% confidence interval: 61-89). Three representative photographs of bacterial adhesion to corneocytes with different colonization status are shown in Figure 2.

A significant difference between strains was only observed for one strain-corneocyte combination. Strain S2 adhered better to corneocytes from D4 compared to the other three strains, including strain S4 isolated from this dog. No bacteria were observed in the negative control slides, confirming absence of bacteria on the collected corneocytes.

\section{Discussion}

This study provides useful knowledge on the variability of $S$. pseudintermedius adherence to corneocytes from dogs with different colonization status. The results indicate a greater adhesion of $S$. pseudintermedius to corneocytes from persistent carriers than intermittent carriers or non-carriers, regardless if the strain was resident or non-resident in the dog from which the corneocytes were collected. To our knowledge, this is the first report showing that adherence of S. pseudintermedius to canine corneocytes is associated with colonization status of the corneocyte donor.

Staphylococcal adhesion to corneocytes is the first step of colonization and a complex biological process involving host and strain factors. A recent study by Moodley et al. [8] showed that $S$. aureus adhered better to corneocytes from colonized pigs than from non-carriers. Similarly, adherence of $S$. aureus to nasal mucosal cells from human $S$. aureus carriers was greater than to cells from non-carriers [9]. In humans, host factors such as polymorphisms in the glucocorticoid receptor gene [10], interleukin 4, complement factor $\mathrm{H}, \mathrm{C}$-reactive genes [11], and distinct histocompatibility antigens [12] have been shown to play an important role in $S$. aureus carriage. Similarly, canine host

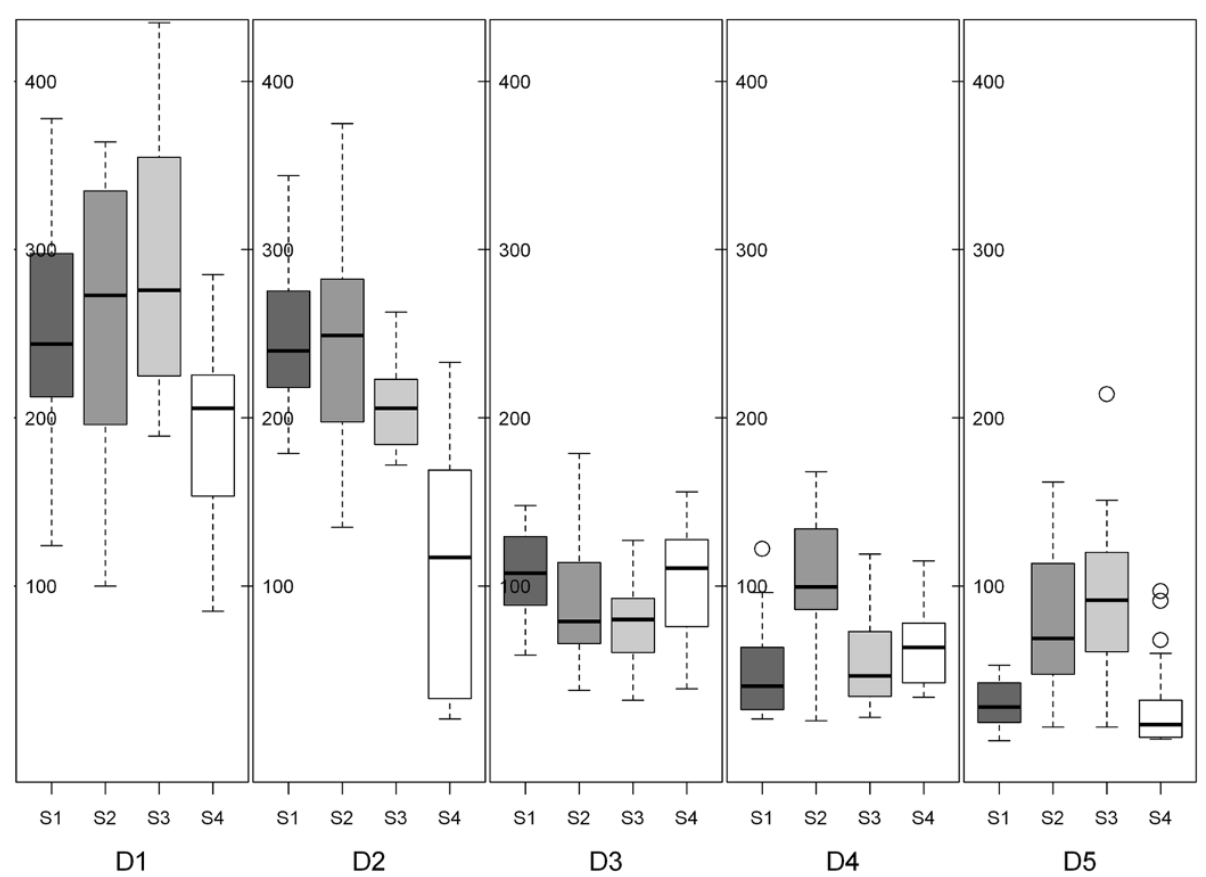

Figure 1 Summary statistics of adherence of S. pseudintermedius to corneocytes. Boxplots illustrating the count of four Staphylococcus pseudintermedius strains (S1-S4) adhering to corneocytes collected from five different dogs (D1-D5). The counts (y-axis) express the summary statistics of number of adhered bacteria per microscopic field for each dog-strain combination. The line within each box represents median; the bottom and top lines of the box represent lower quartile (Q1) and upper quartile (Q3). D1 and D2, persistent carriers; D3 and D4, intermittent carriers; D5, non-carrier; S1-S4, strains from dogs D1-D4. 

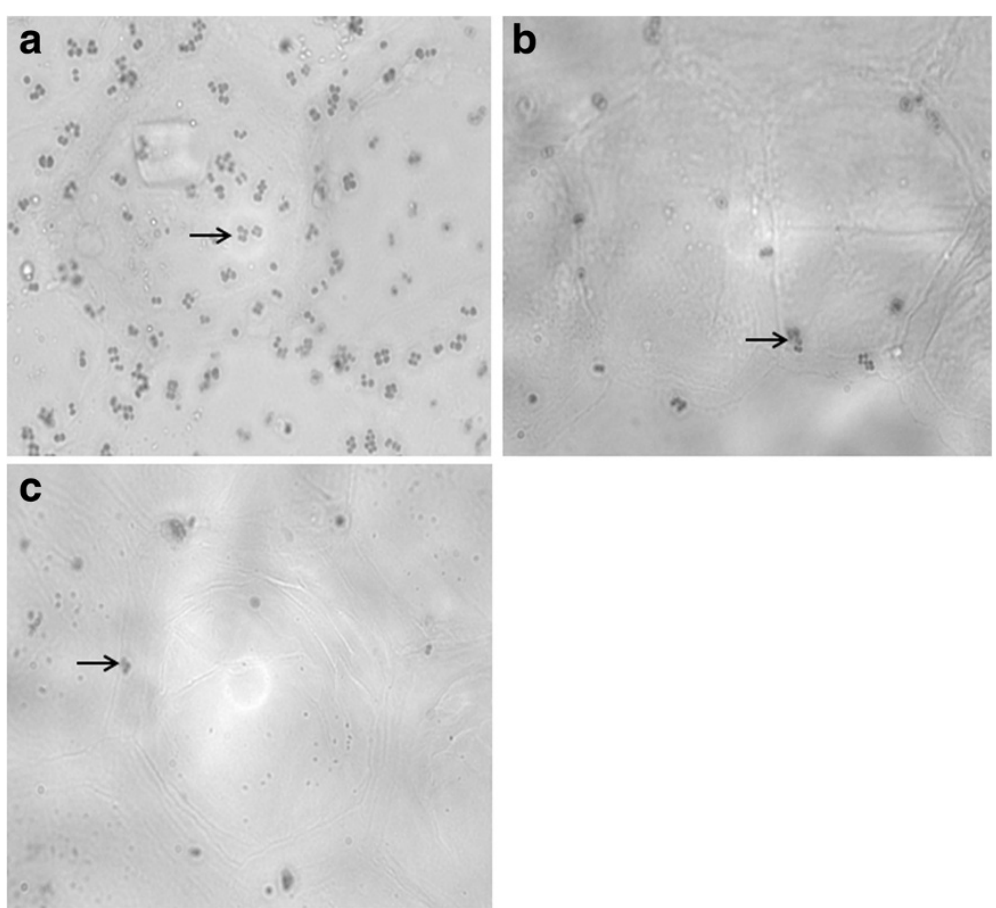

Figure 2 Adherence of Staphylococcus pseudintermedius to corneocytes. Microscopic image ( $\times 1000$ magnification) of S. pseudintermedius strain S2 adhering to corneocytes of: a) a persistent carrier (D1), b) an intermittent carrier (D3), and c) a non-carrier (D5). Arrow signs indicate examples of bacteria adhered to canine corneocytes.

genetic factors may also play a role in S. pseudintermedius carriage. Greater adherence by $S$. pseudintermedius to corneocytes from atopic skin was observed when compared to corneocytes from healthy skin $[5,13]$. This may be due to the changes of skin receptors specific for staphylococci, greater expression of intercellular adhesion and different distribution pattern of fibronectin in atopic skin [13-15].

Breed is an additional host factor that has previously been shown to affect adherence of $S$. pseudintermedius, with greater adherence to corneocytes from breeds such as Boxer and Bull Terriers [3]. As we only studied five dogs belonging to four breeds, this study was not designed to evaluate the role played by this host factor. However, corneocytes from two dogs belonging to the same breed (Labrador Retriever, D1 and D3) displayed different adherence patterns correlating to the colonization status of the two dogs, therefore suggesting that colonization status may play a more important role than breed in $S$. pseudintermedius adherence to corneocytes.

Adhesins are virulence factors that determine the ability of bacteria to adhere to the host tissues [16]. Significant differences in adherence have been reported between strains of S. pseudintermedius with canine corneocytes [4] as well as between strains of $S$. aureus with porcine corneocytes [8]. However, Saijonmaa-Koulumies and Lloyd suggested there is no correlation between virulence and in vitro adherence patterns of S. pseudintermedius [4]. In our study, the strains were genetically distinct and no preferential binding was shown by one strain over the others, with the exception of a particular strain-corneocyte combination (S2-D4). Interestingly this exception did not regard strain and corneocytes from the same dog, showing that preferential binding is not influenced by strain adaptation to individual hosts. Thus, although based on a limited number of dogs, our findings provide a clear indication that $S$. pseudintermedius adherence to canine corneocytes is driven by host factors and only marginally influenced by strain factors. It remains to be determined whether corneocyte adhesion is an important factor for colonization or for initiation of clinical infection. It is not unlikely that mucosal surfaces are the primary colonization site for $S$. pseudintermedius and that skin is repeatedly contaminated by bacteria from mucosal sites. If this hypothesis is true, corneocyte adhesion would be indicative of host predisposition to skin infection rather than colonization. More research is needed to clarify the biological significance of corneocyte adhesion in relation to canine colonization and infection.

S. aureus colonization has been shown to be a risk factor for human infection [17]. Persistent carriers have higher $S$. aureus loads on the nasal mucosa and are more likely to become infected compared to non-carriers [18]. Similarly, persistently colonized dogs harbour higher numbers of $S$. 
pseudintermedius on the oral mucosa [19] and might have a greater risk for infection. The higher frequency of $S$. pseudintermedius colonization in atopic dogs in comparison to healthy dogs [20] suggests that staphylococcal colonization plays an important role in canine pyoderma. Future research is warranted to identify possible host genetic factors predisposing to $S$. pseudintermedius colonization. This information may be used for developing alternative strategies for prevention of staphylococcal infections in dogs. Dog breeds have been created or modified by man to select for specific phenotypic traits such as size, shape, coat colour and behavior. Thus, it is not unrealistic to hypothesize that breeding programmes could be designed to select for dogs with reduced susceptibility to $S$. pseudintermedius colonization.

\section{Competing interests}

The authors declare that they have no competing interests.

\section{Authors' contributions}

NCP, AM, PD and LG: Conceived and designed the experiment. NCP and FL: Performed the experiment. NCP and SSN: Analysed the data. NCP, FL, AM, PD, SSN and LG: Wrote and approved the final manuscript. All authors read and approved the final manuscript.

\section{Acknowledgements}

The study was supported by a grant from Agria och SKKs Forskningsfond for sällskapsdjur (project number N2011-0016) to study host predisposition to S. pseudintermedius colonization in dogs.

\section{Author details}

${ }^{1}$ Department of Veterinary Disease Biology, Faculty of Health and Medical Sciences, University of Copenhagen, Stigbøjlen 4, 1870 Frederiksberg C, Denmark. ${ }^{2}$ Department of Large Animal Science, Faculty of Health and Medical Sciences, University of Copenhagen, Groennegaardsvej 8, 1870 Frederiksberg C, Denmark

Received: 8 May 2013 Accepted: 12 June 2013

Published: 8 July 2013

\section{References}

1. Bannoehr J, Guardabassi L: Staphylococcus pseudintermedius in the dog: taxonomy, diagnostics, ecology, epidemiology and pathogenicity. Vet Dermatol 2012, 23:253-266.

2. Paul NC, Bärgman SC, Moodley A, Nielsen SS, Guardabassi L: Staphylococcus pseudintermedius colonization patterns and strain diversity in healthy dogs: a cross-sectional and longitudinal study. Vet Microbiol 2012, 160:420-427.

3. Forsythe PJ, Hill PB, Thoday KL, Brown J: Use of computerized image analysis to quantify staphylococcal adhesion to canine corneocytes: does breed and body site have any relevance to the pathogenesis of pyoderma? Vet Dermatol 2002, 13:29-36.

4. Saijonmaa-Koulumies LE, Lloyd DH: Adherence of Staphylococcus intermedius to canine corneocytes in vitro. Vet Dermatol 2002, 13:169-176.

5. McEwan NA, Kalna G, Mellor D: A comparison of adherence by four strains of Staphylococcus intermedius and Staphylococcus hominis to canine corneocytes collected from normal dogs and dogs suffering from atopic dermatitis. Res Vet Sci 2005, 78:193-198.

6. Murchan S, Kaufmann ME, Deplano A, de Ryck R, Struelens M, Zinn CE, Fussing V, Salmenlinna S, Vuopio-Varkila J, El Solh N, Cuny C, Witte W, Tassios PT, Legakis N, van Leeuwen W, van Belkum A, Vindel A, Laconcha I, Garaizar J, Haeggman S, Olsson-Liljequist B, Ransjo U, Coombes G, Cookson B: Harmonization of pulsed-field gel electrophoresis protocols for epidemiological typing of strains of methicillin-resistant Staphylococcus aureus: a single approach developed by consensus in 10 European laboratories and its application for tracing the spread of related strains. J Clin Microbiol 2003, 41:1574-1585.
7. Bannoehr J, Ben Zakour NL, Waller AS, Guardabassi L, Thoday KL, van den Broek AH, Fitzgerald JR: Population genetic structure of the Staphylococcus intermedius group: insights into agr diversification and the emergence of methicillin-resistant strains. J Bacterio/ 2007, 189:8685-8692.

8. Moodley A, Espinosa-Gongora C, Nielsen SS, McCarthy AJ, Lindsay JA, Guardabassi L: Comparative host specificity of human- and pigassociated Staphylococcus aureus clonal lineages. PLoS One 2012, 7:e49344.

9. Aly $R$, Shinefield HI, Strauss WG, Maibach HI: Bacterial adherence to nasal mucosal cells. Infect Immun 1977, 17:546-549.

10. van den Akker EL, Nouwen $J$, Melles DC, van Rossum EF, Koper JW, Uitterlinden AG, Hofman A, Verbrugh HA, Pols HA, Lamberts SW, van Belkum A: Staphylococcus aureus nasal carriage is associated with glucocorticoid receptor gene polymorphisms. J Infect Dis 2006, 194:814-818.

11. Emonts $M$, Uitterlinden $A G$, Nouwen JL, Kardys I, Maat MP, Melles DC, Witteman J, Jong PT, Verbrugh HA, Hofman A, Hermans PW, Belkum A Host polymorphisms in interleukin 4, complement factor $\mathrm{H}$, and $\mathrm{C}$ reactive protein associated with nasal carriage of Staphylococcus aureus and occurrence of boils. J Infect Dis 2008, 197:1244-1253.

12. Kinsman OS, McKenna R, Noble WC: Association between histocompatibility antigens (HLA) and nasal carriage of Staphylococcus aureus. J Med Microbiol 1983, 16:215-220.

13. Simou C, Thoday KL, Forsythe PJ, Hill PB: Adherence of staphylococcus intermedius to corneocytes of healthy and atopic dogs: effect of pyoderma, pruritus score, treatment and gender. Vet Dermato/ 2005 16:385-391

14. Cho SH, Strickland I, Boguniewicz M, Leung DY: Fibronectin and fibrinogen contribute to the enhanced binding of staphylococcus aureus to atopic skin. J Allergy Clin Immunol 2001, 108:269-274.

15. Scott DW, Muller WH, Griffin CE: Skin immune system and allergic skin diseases. In Muller and Kirk's small animal dermatology. Philadelphia: W.B. Saunders; 2001:543-666.

16. Coutte L, Alonso S, Reveneau N, Willery E, Quatannens B, Locht C, JacobDubuisson F: Role of adhesin release for mucosal colonization by a bacterial pathogen. J Exp Med 2003, 197:735-742.

17. Wertheim HF, Vos MC, Ott A, van Belkum A, Voss A, Kluytmans JA, van Keulen PH, Vandenbroucke-Grauls CM, Meester MH, Verbrugh HA: Risk and outcome of nosocomial Staphylococcus aureus bacteraemia in nasal carriers versus non-carriers. Lancet 2004, 364:703-705.

18. Nouwen JL, Fieren MW, Snijders S, Verbrugh HA, van Belkum A: Persistent (not intermittent) nasal carriage of Staphylococcus aureus is the determinant of CPD-related infections. Kidney Int 2005, 67:1084-1092.

19. Hartmann FA, White DG, West SE, Walker RD, Deboer DJ: Molecular characterization of Staphylococcus intermedius carriage by healthy dogs and comparison of antimicrobial susceptibility patterns to isolates from dogs with pyoderma. Vet Microbiol 2005, 108:119-131.

20. Fazakerley J, Nuttall T, Sales D, Schmidt V, Carter SD, Hart CA, McEwan NA: Staphylococcal colonization of mucosal and lesional skin sites in atopic and healthy dogs. Vet Dermatol 2009, 20:179-184.

doi:10.1186/1297-9716-44-52

Cite this article as: Paul et al:: In vitro adherence of Staphylococcus pseudintermedius to canine corneocytes is influenced by colonization status of corneocyte donors. Veterinary Research 2013 44:52.

\section{Submit your next manuscript to BioMed Central and take full advantage of:}

- Convenient online submission

- Thorough peer review

- No space constraints or color figure charges

- Immediate publication on acceptance

- Inclusion in PubMed, CAS, Scopus and Google Scholar

- Research which is freely available for redistribution 